\title{
Récurrences virales après un geste opératoire : présentation de 3 cas et revue de littérature
}

\author{
Héloïse Herry*, Anthony Derrien, Julie Lelièvre, Cédric Lansonneur, \\ Sylvie Boisramé-Gastrin
}

UFR Odontologie, Université de Bretagne occidentale, Université européenne de Bretagne, Service d'Odontologie, Pôle Organe des sens, Centre hospitalier régional universitaire, Brest, France

(Reçu le 20 juin 2013, accepté le 26 août 2013)

Mots clés :

herpes virus / récurrence virale / gestes opératoires / chirurgie orale
Key words: herpes virus / viral recurrence / oral operative procedures / oral surgery
Résumé - Introduction : La famille des herpesviridae comprend de nombreux virus dont l'herpes simplex virus (HSV), le virus varicelle-zona (VZV), le cytomégalovirus (CMV), le virus d'Epstein-Barr (EBV). Le HSV, de part sa prévalence élevée (présent chez $90 \%$ de la population adulte), est le plus fréquemment rencontré. Après la primo-infection, le HSV entre dans une phase de latence, puis se réactive sous l'influence de différents facteurs. Trois cas de récurrence herpétique après un geste opératoire de pratique bucco-dentaire quotidienne sont rapportés dans cet article. Cas rapportés : Le premier cas est celui d'un homme de 68 ans qui présente une semaine après l'avulsion de 25 et 27, des vésicules confluentes, non douloureuses, de type herpétique, sur la muqueuse palatine, en regard du site opératoire. Le second cas est celui d'une femme de 89 ans, sans altération de son état général de santé, présentant une semaine après une séance de soins prothétiques (préparation corono-radiculaire et réalisation d'une dent provisoire sur la 16) une multitude de vésicules érosives, non douloureuses, en regard de cette dent. Dans ces deux cas, à des fins pédagogiques, le diagnostic de récurrence herpétique à HSV-1 a été confirmé par analyse biologique. Aucun traitement antiviral n'a été prescrit. Le troisième cas est celui d'une patiente de 55 ans ayant bénéficié d'un retraitement endodontique chez son chirurgien-dentiste traitant. Quelques jours plus tard, elle a présenté un placard génien érythémateux associé à des vésicules douloureuses et des croûtes situées sur le territoire d'innervation du V2. Ces lésions caractéristiques ont fait évoquer un zona qui a été confirmé par la sérologie. La patiente a été hospitalisée et mise sous traitement antiviral. Discussion : Le mécanisme de la réactivation virale est multifactoriel : l'inflammation locale, les micro-traumatismes opératoires, la peur et le stress du patient semblent constituer des facteurs favorisants. Dans certains cas, la récurrence virale est douloureuse : elle nécessite alors un traitement médicamenteux. L'utilisation d'antiseptiques locaux et d'antiviraux (aciclovir et valaciclovir) est conseillée et utilisée en prophylaxie dans les cas de récidive. Les études sur le sujet sont peu nombreuses et toutes s'accordent pour dire que la survenue de ces complications reste rare. Cependant le phénomène existe et il est important de le connaître.

Abstract - Viral recurrences after dental procedures: 3 case reports and review of literature. Introduction: Human herpes viruses are DNA viruses. After the initial infection, some infected people experience sporadic episodes of viral reactivations or outbreaks. These viruses entered in a phase of latency and can reactivate under the influence of different factors and be responsible for recurrences. Report of cases: After dental procedures, the herpes simplex virus is the most frequently encountered. Two cases of viral reactivation and recurrent HSV (one following oral surgery and one after a prosthetic preparation) and one case of VZV viral recurrence (after endodontic retreatment) are reported in this article. For this last case, the treatment required an hospitalization in isolated room to treat pain and viral manifestations in relation with VZV. Discussion: After detailing these few clinical cases, a literature review provides an update on these viral recurrences induced by operative procedures and care. Contributing factors might include trauma to the mouth, emotional stress, and/or a more susceptible immune system. Others factors are described like sunlight, wind, fever, menstruation. Oral herpes can be triggered within about three days of dental work, particularly root canal or tooth extraction. The treatment of these recurrences (still a rare complication) by antiviral (acyclovir, valacyclovir...) is discussed and seems to prove prophylaxis.

\footnotetext{
*Correspondance : heloise87@hotmail.com
} 


\section{Introduction}

En pratique quotidienne bucco-dentaire, les odontologistes sont parfois confrontés à des réactivations virales avec apparition ou non de récurrences cliniques après des gestes opératoires plus ou moins invasifs. C'est la famille des virus herpétiques humains (VHH) qui est la plus concernée par ces récurrences, et surtout l'herpes simplex virus (HSV-1 et 2 ou $\mathrm{VHH}-1$ et 2) ; plus rarement, le virus varicelle-zona (VZV ou $\mathrm{VHH}-3$ ) ou encore le cytomégalovirus (CMV ou VHH-5).

Le but de cet article est de rapporter trois cas et d'effectuer une synthèse de la littérature concernant ces réactivations virales en odontologie afin de mieux comprendre leur mécanisme.

\section{Cas rapportés}

\section{Cas 1}

Un patient âgé de 68 ans a été adressé pour l'avulsion de 25 et 27 . Ce patient avait pour seul antécédent médical une hypertension artérielle essentielle, stabilisée par irbésartan et hydrochlorothiazide (CoAprove ${ }^{\circledR}$ ). Les avulsions ont été réalisées, sans incident au cours de l'intervention. Le patient a été revu une semaine plus tard en contrôle postopératoire, la cicatrisation des sites d'extraction était satisfaisante. Cependant, on observait des érosions post-vésiculeuses confluentes, non douloureuses, situées sur la muqueuse palatine, en regard de la 26 (Fig. 1). À des fins pédagogiques, un prélèvement avec un écouvillon a été réalisé pour une analyse virologique. Les résultats ont conclu à la présence de HSV-1. Le patient a été revu et, sans traitement, la cicatrisation a été obtenue en 15 jours.

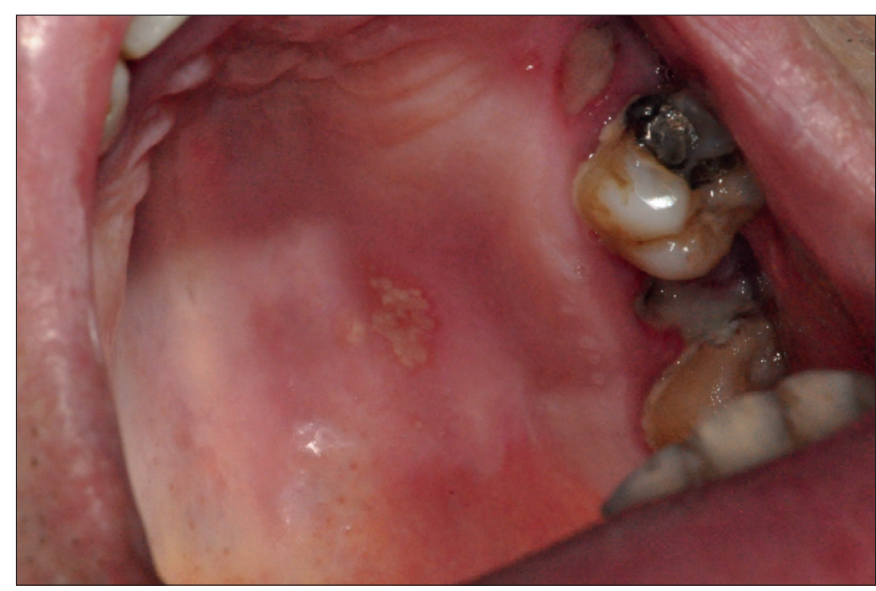

Fig. 1. Cas 1 à $\mathrm{J7}$ : présence d'une plage érythémateuse avec des érosions post-vésiculeuses dans la région palatine postérieure gauche. Fig. 1. Case 1 at D7: erythematous plaque with post-vesicular erosions on the left posterior palatal region.

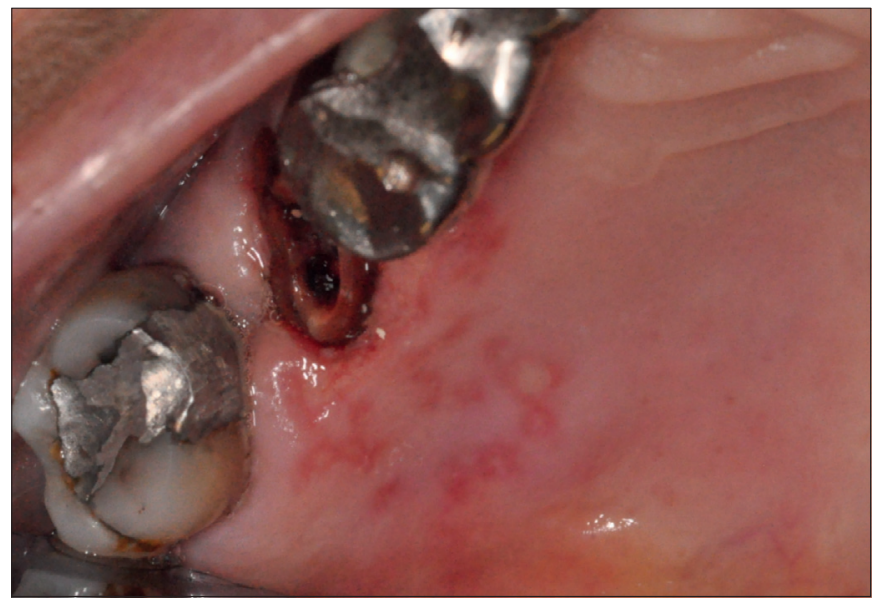

Fig. 2. Cas 2 : aspect de la muqueuse palatine une semaine après une préparation corono-radiculaire sur la 16 : présence d'érosions postvésiculeuses herpétiques confluentes.

Fig. 2. Case 2: palatal mucosa aspect one week after a crown-root preparation. Post-herpetic vesicular confluent érosions.

\section{Cas 2}

Une patiente âgée de 89 ans, sans altération de l'état général de santé, est venue consulter pour la réfection d'une couronne sur la 16 . Lors de la première séance, les soins réalisés (préparation corono-radiculaire de la dent et confection d'une couronne provisoire) ont entraîné un traumatisme de la gencive périphérique (violation de l'espace biologique). Lors de la séance suivante, prévue une semaine plus tard, la patiente présentait en regard de la 16 de multiples érosions post-vésiculeuses (Fig. 2). Même en l'absence de douleurs, l'aspect clinique faisait évoquer une résurgence à HSV-1. La guérison a été obtenue sans traitement médicamenteux.

\section{Cas 3}

Une femme âgée de 55 ans, sans altération de l'état général de santé, s'est présentée aux urgences odontologiques avec une tuméfaction génienne gauche, apparue trois jours après un retraitement endodontique chez son chirurgien-dentiste traitant. L'examen clinique exobuccal a mis en évidence de multiples lésions vésiculeuses unilatérales, localisées dans le territoire du V2. À l'examen endobuccal, des lésions homolatérales sont retrouvées sur le palais, le voile et la lèvre supérieure (Fig. 3). Le diagnostic de zona trigéminal partiel ou zona du V2 a été posé et confirmé par l'examen virologique. La patiente a été hospitalisée. Un traitement antiviral à base $d^{\prime}$ aciclovir par voie intra-veineuse a été prescrit, associé à une détersion cutanée, un traitement antalgique et un traitement à base de prégabaline afin de prévenir les douleurs postzostériennes. De plus, son hospitalisation a nécessité la mise en isolement en raison du risque de contamination par voie 


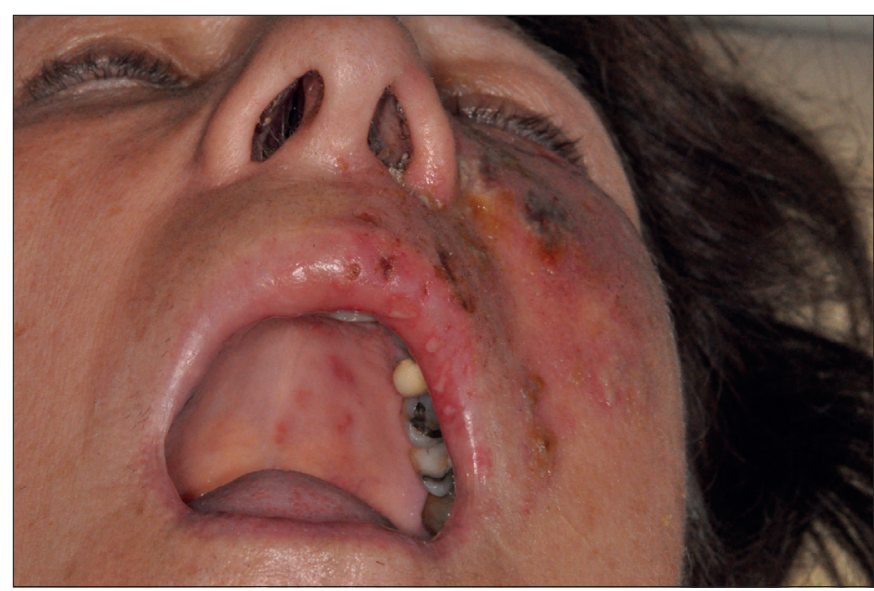

Fig. 3. Cas 3 : présence de nombreuses vésicules et de quelques croûtes sur la joue, l'hémi-lèvre supérieure et l'hémi-palais gauches. Fig. 3. Case 3: many vesicles and some scabs on the left cheek, upper half lip and half palate.

aérienne. Les lésions ont régressé et la guérison était complète après 15 jours d'évolution.

\section{Revue de la littérature et discussion}

Une recherche bibliographique a été menée sur les bases de données Pubmed, Web of sciences et Embase en confrontant les mots clés récurrence virale herpétique et geste opératoire (extraction dentaire, procédure dentaire). L'analyse des titres et des résumés a permis de retrouver des réactivations virales essentiellement herpétiques, avec principalement des récurrences à HSV et exceptionnellement à VZV. Vingt-neuf articles pertinents ont été retenus.

HSV-1 est responsable majoritairement de l'herpès buccal. La prévalence dans la population adulte reste élevée car elle est estimée à 90-95\% [1]. La différenciation entre HSV-1 et HSV-2 est déterminée par des techniques de biologie moléculaire (PCR).

Dans la sphère oro-faciale, après la primo-infection, le virus entre dans une phase de latence plus ou moins longue dans le ganglion trigéminal. Sa réactivation peut se produire à un rythme imprévisible. L'expression clinique de HSV-1 après un acte invasif en pratique bucco-dentaire ne constitue donc pas une infection nosocomiale mais la réactivation de l'infection latente [2]. Cette réactivation apparait aussi éventuellement après $d$ 'autres interventions chirurgicales comme une transplantation cardiaque [3].

Les causes de la réactivation du HSV-1 sont mal connues mais elles semblent associées à différents stimuli : stress, fatigue, hyperthermie, différences importantes de température extérieure, vent, exposition aux ultraviolets, menstruations, immunodépression [4]. Les microtraumatismes comme les blessures labiales, les traumatismes iatrogènes (réalisation d'une préparation coronaire infra-gingivale, présence d'une sonde oro-trachéale, etc. [2]) ou une intervention de chirurgie orale [5-9], constituent également des facteurs déclenchants. Ainsi, plusieurs cas de réactivations de HSV-1 avec apparition de récurrences cliniques après un acte invasif bucco-dentaire ont été décrits. El Hayderi et al. [1] ont rapporté 7 cas de récurrences de HSV-1 après des gestes bucco-dentaires: 5 après extraction de molaires, 1 après extraction d'une incisive et 1 après retraitement endodontique. Williamson a observé un cas de récurrence à HSV-1 à la suite d'une préparation prothétique [5].

La réactivation de HSV-1 entraine une permissivité cutanéo-muqueuse permettant une réplication intra-épidermique ou intra-épithéliale conduisant à la formation de lésions vésiculeuses ; sur la muqueuse, le toit de la vésicule disparait rapidement ce qui laisse place à des érosions post-vésiculeuses. Les lésions cliniques apparaissent en règle générale trois à quatre jours après la réactivation [5].

Un acte chirurgical entrainerait une diminution de l'immunité à médiation cellulaire et humorale, ce qui empêcherait l'organisme de produire des anticorps anti-HSV spécifiques [10]. De plus, les microtraumatismes liés l'acte chirurgical ou opératoire au sens large du terme (préparation prothétique infra-gingivale par exemple) peuvent favoriser la réactivation du virus. Le mécanisme du déclenchement de la réactivation n'est pas totalement élucidé. Cependant, la réactivation implique une modification conformationnelle du génome viral qui passe de sa conformation circulaire hautement associée aux histones à une forme linéaire non nucléosomique. Cette modification serait à l'origine de l'activation de la plus importante protéine transactivatrice VP16 $\left(U_{\mathrm{L}} 48\right)$ nécessaire à l'activation de l'expression des protéines alpha. Il existe, en fait, 3 grandes classes de protéines virales dans le HSV : les protéines alpha issues de gènes précoces qui sont exprimées les premières (ICP4/RS par exemple), les protéines béta (induites par les protéines alpha et impliquées dans la réplication de l'ADN viral), et les protéines gamma (protéines structurales). L'expression des protéines virales VP22/ $\mathrm{U}_{\mathrm{L}} 49$ et ICP4/ $\mathrm{R}_{\mathrm{S}} \mathrm{I}$ entrainent un changement de configuration du génome de l'ADN viral, entretenant ainsi le mécanisme [11]. À partir des tests sérologiques effectués, Hedner et al. retrouvent une augmentation des glycoprotéines virales qui sont des glycopeptides spécifiques (avec principalement $\mathrm{gB}, \mathrm{gC}, \mathrm{gD}$ et ICP 4) [12].

Le mécanisme de réactivation virale est très probablement multifactoriel. Tout d'abord, il a été démontré que la peur et le stress du patient face à une intervention de chirurgie orale augmentent l'excrétion asymptomatique de HSV [13]. Elle peut encore augmenter lors de la lésion de fibres nerveuses par exemple lors d'une extraction dentaire [13]. Au cours des interventions chirurgicales impliquant des branches du nerf trijumeau, la réactivation du HSV surviendrait dans plus de $50 \%$ des cas [10]. Toutefois, l'excrétion du HSV (c'est-à-dire 
la réactivation) ne s'accompagne pas toujours de manifestations cliniques [14, 15]. L'irritation nerveuse secondaire à l'injection de l'anesthésique local pourrait également conduire à la réactivation virale et à la recrudescence de HSV dans les rameaux trigéminaux où le HSV était latent. Ces trois éléments conduiraient à augmenter la charge virale expliquant la sévérité éventuelle de l'éruption.

Lors des réactivations périodiques, le HSV migre de façon antérograde le long des neurones sensitifs jusqu'à la peau ou à la muqueuse et entraînent un épisode d'infection récurrente, même en présence d'une immunité humorale et cellulaire normale. Le site des infections récurrentes tend à rester confiné au dermatome infecté lors de l'infection primaire, mais il peut également y avoir des lésions à distance. La fréquence des récurrences peut dépendre du type du HSV et du site anatomique de l'infection [16]. La sévérité de l'infection primaire, caractérisée par le nombre, l'étendue et la dimension des lésions, favoriserait également les récurrences [17]. Les lésions des kératinocytes, comme on peut en observer en cosmétologie médicale (peeling, resurfaçage au laser abrasif, dermabrasion...), facilitent souvent l'apparition d'une infection cutanée à HSV [14]. Il n'est pas clairement établi s'il existe un lien ou un facteur de risque de cause à effet entre la manipulation et l'étirement des lèvres au cours des soins bucco-dentaires.

On ne retrouve pas dans la littérature de données concernant l'impact des soins bucco-dentaires sur la réactivation du HSV, ni sur son incidence qui est considérée comme rare. Hedner et al. ont effectué une étude afin d'évaluer l'association entre HSV-1 et la survenue d'une alvéolite sèche après l'extraction d'une troisième molaire inférieure [12]. Chez 208 patients, la présence de HSV-1 a été analysée avant et après extraction par des techniques de biologie moléculaire. La proximité anatomique de l'apex du canal du nerf alvéolaire inférieur était évaluée sur la radiographie avant l'extraction. Quinze patients ( $7 \%$ ) ont développé une alvéolite sèche : 11 de ces 15 patients (soit $73 \%$ ) étaient séropositifs pour le HSV-1 contre 7 des 15 patients (soit $47 \%$ ) dans le groupe témoin apparié. Sept des 11 patients séropositifs ont développé une alvéolite sèche associée à une réactivation de HSV-1 confirmée par une augmentation des glycoprotéines virales. Aucune réactivation de HSV1 n'a été observée dans le sérum des sujets contrôles. Les patients atteints d'alvéolite sèche ont montré une fréquence élevée d'antécédents d'herpès labial par rapport aux témoins (64\% contre $33 \%$ ). Aucune différence significative en rapport avec l'intoxication tabagique n'a été retrouvée sur la fréquence de l'herpès labial ou le développement d'une alvéolite sèche. Les auteurs concluent que l'extraction d'une dent de sagesse inférieure pourrait provoquer la réactivation de HSV-1 et le développement d'une alvéolite sèche, surtout en présence d'anticorps, d'une proximité anatomique entre les apex et le nerf alvéolaire inférieur, et de la notion d'antécédents d'herpès labial. À l'opposé, Simon et al. ont analysé les complications après 3818 extractions dentaires : ils n'ont aucun cas de récurrence virale à HSV [18]. Une autre étude cas-témoin comprenant 48 patients séropositifs pour le HSV-1 ayant eu des avulsions de dents de sagesse inférieures et 48 autres patients séropositifs pour le HSV-1 ayant eu des soins conservateurs, montre que la fréquence de HSV-1, confirmée par PCR, était faible $(4,2 \%)$ et non statistiquement significative par rapport au groupe témoin [6].

L'isolement du virus en culture cellulaire reste encore la méthode virologique de référence pour la détection directe du HSV dans les prélèvements. La sensibilité est d'autant plus grande que l'écouvillonnage a été pratiqué sur des lésions récentes. L'écouvillon est ensuite inoculé sur culture cellulaire contenant un milieu (eau, sels minéraux, acides aminés, vitamines, facteurs de croissance, antibiotiques) avec des cellules permissives. Il s'agit de cellules dans lesquelles le cycle viral se déroule en totalité, aboutissant à la lyse cellulaire avec libération des néo-virions. L'effet cytopathogène est habituellement observé en 72 heures. Ces cellules sont soit des cellules Vero (cellules de rein de singe vert), soit des cellules de la lignée MRC-5 (fibroblastes pulmonaires embryonnaires humains...). D'autres méthodes comme la recherche d'antigènes par des techniques ELISA, ont été rapportées pour détecter plus précocement la réplication du HSV [19].

Les 3 cas rapportés dans cet article ont ressenti plus une gêne que des douleurs. Cependant, la plupart des auteurs rapportent une sensation de cuisson, des picotements ou un prurit. Les signes d'accompagnement à type de fébricule ou d'adénopathies restent exceptionnels chez les sujets immunocompétents. El Hayderi et al. signalent des douleurs et des manifestations plus importantes (éruptions douloureuses intéressant la joue, les lèvres, le nez et le menton, associées à une altération de l'état général : fièvre, anorexie, adénopathies) ayant nécessité l'hospitalisation de 2 des 7 patients pour l'administration intra-veineuse d'aciclovir $(5 \mathrm{mg} / \mathrm{kg} / \mathrm{j} \times 8 \mathrm{j}$ ) [1]. Dans le cas rapporté par Williamson, la patiente a noté une sensation de picotements après une prise d'empreinte pour une couronne prothétique, suivie par une douleur pulsatile, puis au $3^{\mathrm{e}}$ jour par des brûlures, et au $4^{\mathrm{e}}$ jour par une douleur sévère irradiant dans la muqueuse buccale [5].

Pour le traitement curatif des récurrences du HSV après un geste opératoire, il n'y a pas de directives précises clairement établies. Le traitement des infections récurrentes du HSV est en général symptomatique et fonction de la gêne et des plaintes du patient. L'utilisation d'antiseptiques locaux et d'antiviraux en traitement topique (aciclovir) peut être intéressante. Chez le patient immunodéprimé, l'aciclovir est prescrit par voie parentérale. Le traitement des infections à HSV, connexes d'une extraction, repose sur un traitement antiviral par voie orale ou intraveineuse, fonction de la gravité clinique. El Hayderi et al. ont traité 5 de leurs patients avec du valaciclovir (Zelitrex $\left.{ }^{\circledR}\right)(500 \mathrm{mg} / \mathrm{j} \times 7 \mathrm{j})$ ou de l'aciclovir (Zovirax ${ }^{\circledR}$ ) $(1000 \mathrm{mg} / \mathrm{j} \times 7 \mathrm{j})$. 
Ce même traitement par aciclovir pendant 5 jours associé à un bain de bouche antiseptique a entrainé une régression significative dès le lendemain dans le cas rapporté par Williamson. Après 3 jours de traitement, la patiente n'avait plus de symptomatologie, hormis lors de la prise d'aliments épicés, et les lésions avaient totalement régressé après 10 jours [5].

Actuellement, la prophylaxie antivirale est réservée aux sujets ayant des antécédents de réactivation virale après un geste opératoire [1]. Pour les deux patients qui nécessitaient des extractions supplémentaires sur les sept ayant eu une récurrence du HSV, El Hayderi et al. ont prescrit un traitement prophylactique à base de valaciclovir $(500 \mathrm{mg}, 2 \mathrm{j}$ avant et $3 \mathrm{j}$ après le geste opératoire) [1]. Williamson a préféré mettre en place une prophylaxie antivirale (aciclovir $200 \mathrm{mg} \times 5 \mathrm{j}$ ) les deux jours précédants tout nouveau geste prothétique chez sa patiente [5]. Par analogie avec le traitement antiviral prophylactique utilisé en dermatologie, le schéma suivant a été proposé par Simon et Matee : valaciclovir $500 \mathrm{mg}$ per os, $2 \mathrm{j}$ avant et $3 \mathrm{j}$ après tout geste dentaire. Ils signalent que le famciclovir ou l'aciclovir peuvent également être prescrits [18].

Le VZV est responsable de la varicelle, sa réactivation du zona. Quelle que soit sa localisation, le zona débute par des signes généraux infectieux. Accompagnée de douleurs, l'éruption cutanéo-muqueuse est unilatérale, intéressant un territoire nerveux (dermatome). Elle est constituée par de multiples vésicules siégeant sur un placard érythémateux [20]. Le contenu clair des vésicules devient rapidement trouble, voire hématique, comme dans le cas rapporté.

Le nerf trijumeau est atteint dans $14 \%$ des cas de zona [21]. Pour ces cas, en raison des symptômes oro-faciaux aigus liés à l'infection virale, l'odontologiste est souvent le professionnel de santé qui est contacté en premier [22]. Le zona du V2 comporte des lésions buccales, affectant souvent d'abord le palais ; elles peuvent constituer une difficulté diagnostique tant qu'elles sont isolées [23]. Ramchandani et al. ont rapporté une complication extrêmement rare se traduisant par un syndrome de Ramsay-Hunt et un empyème sous-dural, provoquée par une récurrence de VZV survenue après avulsions des troisièmes molaires [24]. Certains patients développent une éruption caractéristique plusieurs jours après l'apparition de la paralysie faciale, de sorte que le syndrome de Ramsay-Hunt peut être initialement diagnostiqué à tort comme une paralysie de Bell. Cette dernière se caractérise par une paralysie faciale périphérique aiguë touchant des sujets de tout âge. La plupart des cas sont idiopathiques, tandis que quelques-uns sont secondaires à une infection. L'association entre le HSV-1 et la paralysie de Bell a été rapportée dans les années 1970 mais peu de cas sont survenus après une extraction dentaire [25]. Furuta et al. ont rapporté 8 cas de patients ayant développé une paralysie faciale retardée, survenue après un traitement dentaire ou oro-facial [26]. La réactivation du VZV a été détectée dans $75 \%$ des cas ( 6 patients sur 8 ); dans 3 cas, il y avait un syndrome de Ramsay-Hunt. En l'absence de lésions (zona sine herpete), la réactivation du VZV a été détectée par PCR ou par sérologie. Les résultats suggèrent que la réactivation du VZV est une cause majeure de paralysie faciale retardée après un traitement dentaire ou oro-facial.

Chez des sujets immunodéprimés, ces réactivations peuvent atteindre un degré de gravité plus important. Ainsi Van Gemert et al. ont rapporté le cas d'une femme de 72 ans, atteinte d'une leucémie lymphoïde chronique, adressée pour une éruption cutanée faciale unilatérale après l'extraction de la 16, quelques jours plus tôt. Les examens ont conclu à une récurrence du VZV intéressant le V2 [27]. Le zona facial est une infection grave qui nécessite un traitement précoce. La prise en charge comprend une hospitalisation, avec la mise en place d'un traitement antiviral et analgésique par voie intraveineuse, un traitement antiseptique des lésions et la prescription de gabapentine ou prégabaline afin de prévenir les douleurs postzostériennes, complication du zona compromettant la qualité de vie des patients [28, 29].

\section{Conclusion}

Cet article a eu pour but de montrer, au travers des trois cas rapportés et d'une synthèse de la littérature, que la récurrence des virus herpétiques humains peut être déclenchée par un geste opératoire invasif : chirurgie orale, infiltrations, préparations prothétiques infra-gingivales...

Même si le mécanisme physiopathologique du déclenchement de ces récurrences n'est pas encore clarifié, certains facteurs favorisent leur survenue comme le stress, la fatigue, une hyperthermie, les menstruations, l'immunodépression... Le soleil, le vent ou encore des différences importantes de température extérieure peuvent également constitués des facteurs favorisants.

Des études supplémentaires restent cependant nécessaires pour obtenir des données sur l'incidence de ces récurrences et sur la nécessité ou non d'effectuer un traitement antiviral prophylactique dans certaines situations.

\section{Conflits d'intérêt : aucun}

\section{Références}

1. El Hayderi L, Raty L, Failla V, Caucanas M, Paurobally D, Nikkels AF. Severe herpes simplex virus type-I infections after dental procedures. Medi Oral Patol Oral Cir Bucal 2011;16:e15-8.

2. Fry DE. Herpesviruses: emerging nosocomial pathogens? Surg Infect 2001;2:121-30; discussion 30-2.

3. Tabaee A, Saltman B, Shutter J, Hibshoosh H, Markowitz A. Recurrent oral herpes simplex virus infection presenting as a tongue mass. Oral Surg Oral Med Oral Pathol Oral Radiol Endod 2004;97:376-80. 
4. Yeung-Yue KA, Brentjens MH, Lee PC, Tyring SK. Herpes simplex viruses 1 and 2. Dermatol Clin 2002;20:249-66.

5. Williamson RT. Diagnosis and management of recurrent herpes simplex induced by fixed prosthodontic tissue management: a clinical report. J Prosth Dent 1999;82:1-2.

6. Marques-Silva L, Castro WH, Gomez EL, Guimaraes AL, Silva MS, Gomez RS. The impact of dental surgery on HSV-1 reactivation in the oral mucosa of seropositive patients. J Oral Maxillofac Surg 2007;65:2269-72.

7. Scott DA, Coulter WA, Biagioni PA, O'Neill HO, Lamey PJ. Detection of herpes simplex virus type 1 shedding in the oral cavity by polymerase chain reaction and enzyme-linked immunosorbent assay at the prodromal stage of recrudescent herpes labialis. J Oral Pathol Med 1997;26:305-9.

8. Openshaw H, Bennett HE. Recurrence of herpes simplex virus after dental extraction. J Infect Dis 1982;146:707.

9. Guggenheimer J, Fletcher RD. Traumatic induction of an intraoral reinfection with herpes simplex virus. Report of a case. Oral Surg Oral Med Oral Pathol 1974;38:546-9.

10. Porteous C, Bradley JA, Hamilton DN, Ledingham IM, Clements GB, Robinson CG. Herpes simplex virus reactivation in surgical patients. Crit Care Med 1984;12:626-8.

11. Wysoka J, Herr W. The herpes simplex virus VP16-induced complex: the makings of a regulatory switch. Trends Biochem Sci 2003;28:294-304.

12. Hedner E, Vahlne A, Kahnberg KE, Hirsch JM. Reactivated herpes simplex virus infection as a possible cause of dry socket after tooth extraction. J Oral Maxillofac Surg 1993;51:370-6; discussion 77-8.

13. Hyland PL, Coulter WA, Abu-Ruman I, Fulton CR, O'Neill HJ, Coyle PV, Lamey P-J. Asymptomatic shedding of HSV-1 in patients undergoing oral surgical procedures and attending for noninvasive treatment. Oral Dis 2007;13:414-8.

14. da Silva LM, Guimaraes AL, Victoria JM, Gomes CC, Gomez RS. Herpes simplex virus type 1 shedding in the oral cavity of seropositive patients. Oral Dis 2005;11:13-6.

15. Knaup B, Schunemann S, Wolff MH. Subclinical reactivation of herpes simplex virus type 1 in the oral cavity. Oral Microbiol Immunol 2000;15:281-3.
16. Lafferty WE, Coombs RW, Benedetti J, Critchlow C, Corey L. Recurrences after oral and genital herpes simplex virus infection. Influence of site of infection and viral type. New Engl J Med 1987;316:1444-9.

17. Benedetti J, Corey L, Ashley R. Recurrence rates in genital herpes after symptomatic first-episode infection. Ann Intern Med 1994;121:847-54.

18. Simon E, Matee M. Post-extraction complications seen at a referral dental clinic in Dar es Salaam, Tanzania. Int Dent J 2001;51:273-6.

19. Chanzy B, Braig S, Morand P. Debates and updates regarding the virologic diagnosis of herpes. Pathol Biol (Paris) 2002;50: 419-24.

20. Kuffer R, Lombardi T, Husson-Bui C, Courrier B, Samson J. Infections virales (pp. 297-312). In: La muqueuse buccale : de la clinique au traitement. Editions Med'Com, Paris, 2009.

21. Hope-Simpson RE. The nature of herpes zoster: a long-term study and a new hypothesis. Proc R Soc Med 1965;58:9-20.

22. Birek C. Herpesvirus-induced diseases: oral manifestations and current treatment options. J Calif Dent Assoc 2000;28:911-21.

23. Ahossi V, Boisramé-Gastrin S, Larras P, Tazi M, Perrin D. Zona trigéminal : difficulté diagnostique et complications odontologiques. Actual Odonto-Stomatol 2004;228:317-25.

24. Ramchandani PL, Sabesan T, Peters WJ. Subdural empyema and herpes zoster syndrome (Hunt syndrome) complicating removal of third molars. Br J Oral Maxillofac Surg 2004;42:55-7.

25. Owsley D, Goldsmith JP. Bell's palsy following primary tooth extraction. A case report. NY State Dent J 2012;78:32-3.

26. Furuta $Y$, Ohtani F, Fukuda S, Inuyama Y, Nagashima K. Reactivation of varicella-zoster virus in delayed facial palsy after dental treatment and oro-facial surgery. J Med Virol 2000;62:42-5.

27. van Gemert JT, Koole R. An unusual skin disorder after tooth extraction. Nede Tijdschr Tandheelkd 2007;114:98-103.

28. Makharita MY, Amr YM, El-Bayoumy Y. Effect of early stellate ganglion blockade for facial pain from acute herpes zoster and incidence of postherpetic neuralgia. Pain Physician 2012;15: 467-74.

29. Andrei G, Snoeck R. Advances in the treatment of varicella-zoster virus infections. Adv Pharmacol 2013;67:107-68. 